\title{
1-(2-Chlorobenzyloxy)-3-[1,2,3]triazol-1-yl-propan-2-ol Derivatives: Synthesis, Characterization, and DFT-Based Descriptors Analysis
}

\author{
Eloisa Román-Maldonado, ${ }^{1}$ Horacio Reyes, ${ }^{2}$ Miguel A. Sanchez-Carmona, ${ }^{1}$ \\ Nelly González-Rivas, ${ }^{1}$ and Erick Cuevas-Yañez ${ }^{1}$ \\ ${ }^{1}$ Centro Conjunto de Investigación en Química Sustentable UAEM-UNAM, Universidad Autónoma del Estado de México, \\ Carretera Toluca-Atlacomulco Km 14.5, 50200 Toluca, MEX, Mexico \\ ${ }^{2}$ División de Ingeniería Química, Instituto de Estudios Superiores de Jocotitlán, Carretera Toluca-Atlacomulco Km 44.8, \\ Ejido de San Juan y San Agustin, Jocotitlán, MEX, Mexico
}

Correspondence should be addressed to Erick Cuevas-Yañez; ecuevasy@uaemex.mx

Received 14 December 2016; Accepted 13 February 2017; Published 13 March 2017

Academic Editor: Anton Kokalj

Copyright (C) 2017 Eloisa Román-Maldonado et al. This is an open access article distributed under the Creative Commons Attribution License, which permits unrestricted use, distribution, and reproduction in any medium, provided the original work is properly cited.

A novel series of 1-(2-chlorobenzyloxy)-3-[1,2,3]triazol-1-yl-propan-2-ol derivatives was designed and synthesized using copper catalyzed alkyne-azide cycloaddition in the key step. Theoretical investigation of molecular and electronic properties by means of global and local reactivity indexes of the synthetized compounds was carried out, using DFT (Density Functional Theory) at $\mathrm{PBEPBE} / 6-31++\mathrm{G}^{* *}$ level.

\section{Introduction}

Depression and similar psychological disorders affect about 350 million people worldwide and represent one of the leading causes of disability and a major contributor to the burden of suicide and ischemic heart diseases $[1,2]$. This illness is associated with a decreased production of serotonin in conjunction with a decreased sensitivity of postsynaptic receptors to available serotonin [3]. Therefore, one of the most extended current therapies for this kind of diseases involve the use of commercially available drugs which work as selective serotonin reuptake inhibitors (SSRI) such as fluoxetine among other analogous compounds $[4,5]$. Although all these compounds are effective as antidepressants, some side effects are observed during their use. This reason has motivated the design and development of diverse molecules, which display good reuptake inhibiting activity [6-8].

A particular molecule that has attracted us is a derivative from chlorobenzyloxy-3,6-dihydropyridinyl propan-2ol named DJLDU-3-79, 1 [9]. This compound was designed from a hybridization of two active reuptake inhibitory molecules in order to improve the above-mentioned properties. However, a synthetic challenge in the preparation of this molecule is the tetrahydropyridine moiety prepared from 4-piperidone analogs, which in turn are obtained through several reaction steps $[10,11]$. In this regard, we propose a synthesis of a novel family of compounds 2 analogs to molecule 1 based on a substitution of tetrahydropyridine ring by 1,2,3-triazole which is easily synthesized by copper catalyzed alkyne-azide cycloaddition (CuAAC), the most powerful click reaction (Scheme 1).

On the other hand, DFT-based methods have already proven to be useful in determining molecular structure of organic compounds, as well as in elucidating their electronic structure and reactivity [12]. There are two kinds of DFTbased reactivity indexes: global and local quantities. The global parameters measure the electronic features of the molecules as a whole and the local quantity reflects the reactivity of particular regions or atoms. The properties calculated in this study have been successfully applied in to explain the reactivity of organic and inorganic molecules, proteins, solids, and others [13]. 

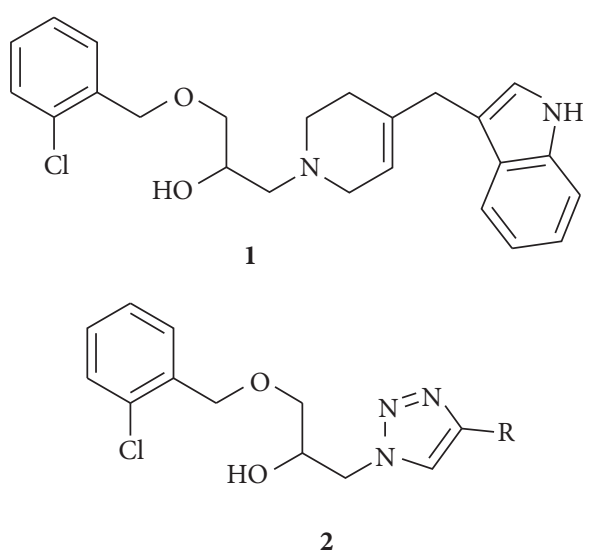

Scheme 1: Structure of DJLDU-3-79 (1) and general structure for molecules 2 proposed in this work.

Several studies have confirmed closed relationships between the biological performance of a family of compounds and their chemical reactivity. The pharmacokinetic and pharmacodynamic properties of SSRI molecules are strongly influenced by their interactions to the body proteins [9]. Therefore, the electron transfer properties in proteinligand binding could be studied by using DFT reactivity indexes. To accomplish this goal, the use of global chemical reactivity parameters, chemical potential $(I)$, electron affinity $(A)$, chemical potential $(\mu)$, softness $(S)$, and electrophilicity $(\omega)$, could aid the understanding of the interaction process between SSRI and biological molecules. Also, the local parameters could give some light about which molecular moieties are most involved in the binding structure

This report summarizes our most recent findings in the click chemistry area aiming to search and develop new molecules with high SSRI activity; also the theoretical characterization of the obtained molecules was carried out.

\section{Experimental Section}

2.1. Synthesis. The starting materials were purchased from Aldrich Chemical Co. and were used without further purification. Solvents were distilled before use. Silica plates of $0.20 \mathrm{~mm}$ thickness were used for thin layer chromatography. Melting points were determined with a Krüss Optronic melting point apparatus and they are uncorrected. ${ }^{1} \mathrm{H}$ and ${ }^{13} \mathrm{C}$ NMR spectra were recorded using a Bruker AVANCE 300 ; the chemical shifts $(\delta)$ are given in ppm relative to TMS as internal standard (0.00). For analytical purposes the mass spectra were recorded on a Shimadzu GCMS-QP2010 Plus in the EI mode, $70 \mathrm{eV}, 200^{\circ} \mathrm{C}$ via direct inlet probe. Only the molecular and parent ions $(\mathrm{m} / \mathrm{z})$ are reported. IR spectra were recorded on a Bruker TENSOR 27 FT instrument.

2.1.1. 2-Chlorophenyl-methanol (4). A solution of 2-chlorobenzaldehyde (1.125 mL, $1.40 \mathrm{~g}, 10.03 \mathrm{mmol})$ in ethanol $(15 \mathrm{~mL})$ was added to a suspension of sodium borohydride $(0.189 \mathrm{~g}$, $4.97 \mathrm{mmol})$ in absolute ethanol $(6 \mathrm{~mL})$ maintaining the temperature below $25^{\circ} \mathrm{C}$. The resulting mixture was heated at $50^{\circ} \mathrm{C}$ during $1.5 \mathrm{~h}$. The solvent was removed in vacuo, and water $(20 \mathrm{~mL})$ was added; the mixture was acidified with diluted $\mathrm{HCl}(10 \%)$ to $\mathrm{pH}=5$. The product was extracted with $\mathrm{CH}_{2} \mathrm{Cl}_{2}(3 \times 15 \mathrm{~mL})$, the organic phases were joined and dried over $\mathrm{Na}_{2} \mathrm{SO}_{4}$, and the solvent was removed in vacuo to yield a white solid (1.27 g, 90\%), which was used without additional purification, m.p. $50^{\circ} \mathrm{C}$; ${ }^{1} \mathrm{H}$ NMR $\left(300 \mathrm{MHz} \mathrm{CDCl}_{3}\right) \delta 7.49$ (dd, $J=7.3,2.0 \mathrm{~Hz}, 1 \mathrm{H}), 7.37$ (dd, $J=7.5,1.8 \mathrm{~Hz}, 1 \mathrm{H}), 7.32-7.20$ $(\mathrm{m}, 2 \mathrm{H}), 4.79(\mathrm{~d}, J=6.0 \mathrm{~Hz}, 1 \mathrm{H}), 1.69(\mathrm{~s}, 1 \mathrm{H}) ;{ }^{13} \mathrm{C} \mathrm{NMR}$ $\left(75 \mathrm{MHz}, \mathrm{CDCl}_{3}\right) \delta 137.1,131.6,128.2,127.7,127.6,125.9,61.7$; IR (ATR, $\left.\mathrm{cm}^{-1}\right): 3302,3216,2912,2859,1439 ; \mathrm{MS}[\mathrm{EI}+] \mathrm{m} / z$ (\%): $142[\mathrm{M}]^{+}(1), 107\left[\mathrm{C}_{7} \mathrm{H}_{7} \mathrm{O}\right]^{+}(35), 77\left[\mathrm{C}_{6} \mathrm{H}_{5}\right]^{+}(100), 51$ $\left[\mathrm{C}_{4} \mathrm{H}_{3}\right]^{+}(91)$.

2.1.2. 2-(2-Chloro-benzyloxymethyl)-oxirane (5). A mixture of $40 \%$ aqueous solution of $\mathrm{NaOH}(6.5 \mathrm{~mL})$ (2-chlorophenyl)-methanol $4(0.71 \mathrm{~g}, 5 \mathrm{mmol})$ and tetrabutylammonium iodide $(0.09 \mathrm{~g}, 0.25 \mathrm{mmol})$ was vigorously stirred at room temperature for $24 \mathrm{~h}$. The mixture was cooled to $0^{\circ} \mathrm{C}$ and epichlorohydrin $(1.55 \mathrm{~mL}, 1.83 \mathrm{~g}$, and $19.9 \mathrm{mmol})$ was added dropwise. After completion, the reaction mixture was stirred at $0^{\circ} \mathrm{C}$ for 30 minutes and at room temperature overnight. The mixture was poured into ice cold water $(20 \mathrm{~mL})$. The product was extracted with $\mathrm{CH}_{2} \mathrm{Cl}_{2}(3 \times 15 \mathrm{~mL})$, the organic phases were joined and dried over $\mathrm{Na}_{2} \mathrm{SO}_{4}$, and the solvent was removed in vacuo to yield a pale yellow oil (1.27 g, 90\%), which was used without additional purification; ${ }^{1} \mathrm{H}$ NMR $\left(300 \mathrm{MHz}, \mathrm{CDCl}_{3}\right) \delta 7.50(\mathrm{dd}, J=7.4,2.1 \mathrm{~Hz}, 1 \mathrm{H})$, 7.35 (dd, $J=7.4,1.8 \mathrm{~Hz}, 1 \mathrm{H}), 7.31-7.19(\mathrm{~m}, 2 \mathrm{H}), 4.68$ (d, $J=$ $3.9 \mathrm{~Hz}, 2 \mathrm{H}), 3.49-3.87$ (dd, $J=11.4,5.8 \mathrm{~Hz}, 2 \mathrm{H}), 3.27-3.18$ (m, $1 \mathrm{H}), 2.65-2.82(\mathrm{dd}, J=5.0,2.7 \mathrm{~Hz}, 2 \mathrm{H}) ;{ }^{13} \mathrm{C} \mathrm{NMR}(75 \mathrm{MHz}$, $\left.\mathrm{CDCl}_{3}\right) \delta 135.4,132.6,129.0,128.7,128.5,126.5,71.1,69.9,50.5$, 43.9; IR (ATR, cm ${ }^{-1}$ ): 3061, 2998, 2923, 2864, 1250, 1096; MS [EI+] $m / z(\%): 198[\mathrm{M}]^{+}(5), 56\left[\mathrm{C}_{3} \mathrm{H}_{5} \mathrm{O}\right]^{+}$(100). HRMS (EI): calcd. For $\mathrm{C}_{10} \mathrm{H}_{11} \mathrm{ClO}_{2}$ : 198.0448; found: 198.0442 .

2.1.3. 1-Azido-3-(2-chloro-benzyloxy)-propan-2-ol (6). 2-(2Chloro-benzyloxymethyl)-oxirane $5(0.965 \mathrm{~g}, 4.86 \mathrm{mmol})$, $\mathrm{NH}_{4} \mathrm{Cl}(0.529 \mathrm{~g}, 9.89 \mathrm{mmol})$, and $\mathrm{NaN}_{3}$ (2.652 g, $\left.40.8 \mathrm{mmol}\right)$ were added successively to a mixture of $\mathrm{MeOH}(8 \mathrm{~mL})$ and $\mathrm{H}_{2} \mathrm{O}(1 \mathrm{~mL})$ and the reaction mixture was stirred at room temperature for $24 \mathrm{~h}$. The solvent was removed in vacuo and the product was extracted with $\operatorname{AcOEt}(3 \times 15 \mathrm{~mL})$; the organic phases were joined and dried over $\mathrm{Na}_{2} \mathrm{SO}_{4}$ and the solvent was removed in vacuo to yield a pale yellow oil $(1.2981 \mathrm{~g}, 74.12 \%)$, which was used without additional purification; ${ }^{1} \mathrm{H} \mathrm{NMR}\left(300 \mathrm{MHz}, \mathrm{CDCl}_{3}\right) \delta 7.40$ (dd, $J$ $=7.2,2.2 \mathrm{~Hz}, 1 \mathrm{H}), 7.35-7.30(\mathrm{~m}, 1 \mathrm{H}), 7.29-7.16(\mathrm{~m}, 2 \mathrm{H})$, $4.60(\mathrm{~s}, 2 \mathrm{H}), 3.94$ (ddd, $J=10.8,5.9,4.9 \mathrm{~Hz}, 2 \mathrm{H}), 3.54$ (s, broad, $1 \mathrm{H}), 3.52-3.34$ (ddd, $J=5.4,2.3,1.3 \mathrm{~Hz}, 2 \mathrm{H}$ ), $3.22(\mathrm{~s}, 1 \mathrm{H}) ;{ }^{13} \mathrm{C} \mathrm{NMR}\left(75 \mathrm{MHz}, \mathrm{CDCl}_{3}\right) \delta 135.3,133.2$, $129.4,129.4,129.1,126.9,71.85,70.6,69.6,53.5$; IR (ATR, $\left.\mathrm{cm}^{-1}\right): 3426,2097,1092,751 ; \mathrm{MS}[\mathrm{EI}+] \mathrm{m} / z(\%): 241[\mathrm{M}]^{+}$

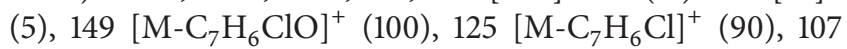
$\left[\mathrm{M}-\mathrm{C}_{3} \mathrm{H}_{6} \mathrm{~N}_{3} \mathrm{O}\right]+(90), 89\left[\mathrm{M}-\mathrm{C}_{2} \mathrm{H}_{4} \mathrm{~N}_{3} \mathrm{O}\right]^{+}(60), 43\left[\mathrm{M}-\mathrm{N}_{3}\right]^{+}$ (65). HRMS (EI): calcd. For $\mathrm{C}_{10} \mathrm{H}_{12} \mathrm{ClN}_{3} \mathrm{O}_{2}$ : 241.0618; found: 241.0622 . 
2.2. General Procedure for the Synthesis of 1-(2-Chlorobenzyloxy)-3-[1,2,3]triazol-1-yl-propan-2-oles. The appropriate alkyne $(1 \mathrm{~mol})$ was added in one portion to a solution of 1-azido-3-(2-chloro-benzyloxy)-propan-2-ol 6 (0.241g, $1 \mathrm{mmol})$, CuI (0.009 g, $0.05 \mathrm{mmol})$, and DIPEA $(0.17 \mathrm{~mL}$, $0.129 \mathrm{~g}, 1 \mathrm{mmol})$ in $\mathrm{CH}_{2} \mathrm{Cl}_{2}(15 \mathrm{~mL})$. The resulting mixture was stirred at room temperature for $24 \mathrm{~h}$. An aqueous solution of $2 \%$ EDTA $(30 \mathrm{~mL})$ was added and the stirring was continued for additional $24 \mathrm{~h}$. The product was extracted with $\mathrm{CH}_{2} \mathrm{Cl}_{2}(3 \times 15 \mathrm{~mL})$, the organic phases were joined and dried over $\mathrm{Na}_{2} \mathrm{SO}_{4}$, the solvent was removed in vacuo, and the final product was purified by crystallization.

2.2.1. 1-(2-Chlorobenzyloxy)-3-(4-phenyl-[1,2,3]triazol-1-yl)propan-2-ol (7). White solid; yield: $85 \%$; $\mathrm{mp} 58^{\circ} \mathrm{C} ;{ }^{1} \mathrm{H}$ NMR $\left(300 \mathrm{MHz}, \mathrm{CDCl}_{3}\right) \delta 7.86(\mathrm{~s}, 1 \mathrm{H}), 7.78-7.65(\mathrm{~m}, 2 \mathrm{H}), 7.47-7.30$ (m, 5H), $7.32(\mathrm{~s}, 1 \mathrm{H}), 7.30-7.20(\mathrm{~m}, 2 \mathrm{H}), 4.65(\mathrm{~s}, 2 \mathrm{H}), 4.64-4.57$ (m), $4.49(\mathrm{dd}, J=8.7,7.0 \mathrm{~Hz}, 1 \mathrm{H}), 4.48-4.36(\mathrm{~m}, 1 \mathrm{H}), 3.59-3.52$ (m, $1 \mathrm{H}), 3.44-3.37(\mathrm{~m}, 1 \mathrm{H}), 2.62(\mathrm{~s}$, broad, $1 \mathrm{H}) ;{ }^{13} \mathrm{C}$ NMR $\left(75 \mathrm{MHz}, \mathrm{CDCl}_{3}\right) \delta 135.3,133.2,129.4,129.4,129.1,126.9,71.8$, 70.6, 69.6, 53.5; IR (ATR, $\mathrm{cm}^{-1}$ ): 3227, 3061, 2866, 1440, 750; MS [EI+] $\mathrm{m} / z(\%): 343[\mathrm{M}]^{+}(5), 125\left[\mathrm{C}_{7} \mathrm{H}_{6} \mathrm{Cl}\right]^{+}(100)$. HRMS (EI): calcd. For $\mathrm{C}_{18} \mathrm{H}_{18} \mathrm{ClN}_{3} \mathrm{O}_{2}$ : 343.1088; found: 343.1091 .

2.2.2. 1-(2-Chlorobenzyloxy)-3-(4-p-tolyloxymethyl-[1,2,3]triazol-1-yl)-propan-2-ol (8). White solid; yield: $99 \%$; $\mathrm{mp} 25^{\circ} \mathrm{C}$; ${ }^{1} \mathrm{H}$ NMR $\left(300 \mathrm{MHz}, \mathrm{CDCl}_{3}\right) \delta 7.70(\mathrm{~s}, 1 \mathrm{H}), 7.46(\mathrm{dd}, J=7.6$, $1.7 \mathrm{~Hz}, 1 \mathrm{H}), 7.41(\mathrm{dd}, J=3.8,1.7 \mathrm{~Hz}, 1 \mathrm{H}), 7.27(\mathrm{dd}, J=12.2$, $1.8 \mathrm{~Hz}, 1 \mathrm{H}), 7.20-7.13(\mathrm{~m}, 1 \mathrm{H}), 7.05(\mathrm{~d}, J=8.5 \mathrm{~Hz}, 2 \mathrm{H}), 6.87(\mathrm{~d}$, $J=2.3 \mathrm{~Hz}, 1 \mathrm{H}), 6.83(\mathrm{~d}, J=2.1 \mathrm{~Hz}, 1 \mathrm{H}), 5.08(\mathrm{~s}, 2 \mathrm{H}), 4.57(\mathrm{~s}$, $2 \mathrm{H}$ ), $4.52-4.36$ (dddd, $J=13.9,14.0,3.8,7.1 \mathrm{~Hz}, 2 \mathrm{H}$ ), 4.20 (dd, $J=9.2,5.2 \mathrm{~Hz}, 1 \mathrm{H}), 3.53-3.46$ (ddd, $J=9.7,5.4,4.9 \mathrm{~Hz}, 2 \mathrm{H}$ ), 2.49 (s, broad, $1 \mathrm{H}), 2.25(\mathrm{~s}, 3 \mathrm{H}) ;{ }^{13} \mathrm{C}$ NMR $\left(75 \mathrm{MHz}, \mathrm{CDCl}_{3}\right)$ $\delta 155.1,143.0,134.7,134.2,132.3,129.5,128.5,128.5,127.9,125.8$, 123.4, 113.8, 70.6, 69.5, 68.1, 60.93, 52.1, 19.5; IR (ATR, $\left.\mathrm{cm}^{-1}\right)$ : 3312, 2925, 2860, 1269, 1120; MS [EI+] $m / z(\%): 387[\mathrm{M}]^{+}(5)$, $125\left[\mathrm{C}_{7} \mathrm{H}_{6} \mathrm{Cl}\right]^{+}(100)$. HRMS (EI): calcd. For $\mathrm{C}_{20} \mathrm{H}_{22} \mathrm{ClN}_{3} \mathrm{O}_{3}$ : 387.1350; found: 387.1351 .

2.2.3. 1-(2-Chlorobenzyloxy)-3-[4-(4-methoxy-phenoxymeth$y l)$-[1,2,3]triazol-1-yl]-propan-2-ol (9). White solid; yield: 46\%; mp $76^{\circ} \mathrm{C} ;{ }^{1} \mathrm{H}$ NMR $\left(300 \mathrm{MHz}, \mathrm{CDCl}_{3}\right) \delta 7.78(\mathrm{~s}, 1 \mathrm{H})$, $7.75-7.68(\mathrm{~m}, 2 \mathrm{H}), 7.39(\mathrm{dd}, J=10.7,4.2 \mathrm{~Hz}, 2 \mathrm{H}), 7.31-7.20(\mathrm{~m}$, $2 \mathrm{H}), 6.95(\mathrm{~d}, J=8.4 \mathrm{~Hz}, 2 \mathrm{H}), 4.65(\mathrm{~s}, 1 \mathrm{H}), 4.60-4.46$ (dddd, $J$ $=14.2,3.6,14.0,6.8 \mathrm{~Hz}, 2 \mathrm{H}), 4.36-4.26(\mathrm{~m}, 1 \mathrm{H}), 3.84(\mathrm{~s}, 3 \mathrm{H})$, $3.63-3.53$ (ddd $J=9.8,4.9,9.7,5.7 \mathrm{~Hz}, 2 \mathrm{H}$ ), 2.04 (s, broad, $1 \mathrm{H}$ ); ${ }^{13} \mathrm{C}$ NMR $\left(75 \mathrm{MHz}, \mathrm{CDCl}_{3}\right) \delta 159.6,147.5,135.0,133.5 \mathrm{de} \mathrm{C} 3$, $129.6,129.6,129.3,126.9,126.9,126.9,120.4,114.2,71.3,70.8$, 69.3, 55.3, 52.9; IR (ATR, $\left.\mathrm{cm}^{-1}\right): 3256,3141,2958,2924,2854$, 1360, 1249; MS [EI+] $m / z(\%): 403[\mathrm{M}]^{+}(28), 125\left[\mathrm{C}_{7} \mathrm{H}_{6} \mathrm{Cl}\right]^{+}$ (100).

2.2.4. 1-(2-Chlorobenzyloxy)-3-(4-p-tolyl-[1,2,3]triazol-1-yl)propan-2-ol (10). White solid; yield: $51 \%$; $\mathrm{mp} 120^{\circ} \mathrm{C} ;{ }^{1} \mathrm{H}$ NMR $\left(300 \mathrm{MHz}, \mathrm{CDCl}_{3}\right) \delta 7.81(\mathrm{~s}, 1 \mathrm{H}), 7.64-7.55(\mathrm{~m}, 2 \mathrm{H})$, $7.42(\mathrm{dd}, J=6.4,2.9 \mathrm{~Hz}, 1 \mathrm{H}), 7.29-7.14(\mathrm{~m}, 5 \mathrm{H}), 4.64(\mathrm{~s}, 2 \mathrm{H})$, $4.58-4.45$ (dddd, $J=14.0,7.2,3.8,4.8 \mathrm{~Hz}, 2 \mathrm{H}), 4.44-4.38$ (m, $1 \mathrm{H}), 3.61-3.57(\mathrm{~m}, 2 \mathrm{H}), 2.36(\mathrm{~s}, 3 \mathrm{H}), 1.26(\mathrm{~s}$, broad, $1 \mathrm{H}) ;{ }^{13} \mathrm{C}$
NMR $\left(75 \mathrm{MHz}, \mathrm{CDCl}_{3}\right) \delta 155.1,143.0,134.7,134.22,132.3$, $129.5,128.5,128.8,127.9,125.8,123.4,113.8,70.6,69.5,68.1$, 60.9, 52.1, 19.5; IR (ATR, $\left.\mathrm{cm}^{-1}\right)$ : 3392, 2918, 2865, 1442, 1107; MS [EI+] $m / z(\%): 357[\mathrm{M}]^{+}(5), 125\left[\mathrm{C}_{7} \mathrm{H}_{6} \mathrm{Cl}\right]^{+}(100)$. HRMS (EI): calcd. For $\mathrm{C}_{19} \mathrm{H}_{20} \mathrm{ClN}_{3} \mathrm{O}_{2}$ : 357.1244; found: 357.1247 .

2.2.5. 2-\{1-[3-(2-Chlorobenzyloxy)-2-hydroxy-propyl]-1H-[1,2, 3]triazol-4-ylmethyl\}-2-methyl-cyclopentane-1,3-dione (11). Colorless oil; yield: $66 \% ;{ }^{1} \mathrm{H}$ NMR $\left(300 \mathrm{MHz}, \mathrm{CDCl}_{3}\right) \delta 7.44$ $(\mathrm{s}, 1 \mathrm{H}), 7.48-7.41(\mathrm{~m}, 1 \mathrm{H}), 7.36-7.31(\mathrm{~m}, 1 \mathrm{H}), 7.26-7.19(\mathrm{~m}, 2 \mathrm{H})$, 4.60 (s, 2H), 4.46-4.29 (dddd, $J=14.0,13.9,7.3,3.6 \mathrm{~Hz}, 2 \mathrm{H}$ ), $4.14(\mathrm{dq}, J=9.4,5.3 \mathrm{~Hz}, 1 \mathrm{H}), 3.50(\mathrm{~d}, J=5.2 \mathrm{~Hz}, 2 \mathrm{H}), 3.05(\mathrm{~s}$, $2 \mathrm{H}), 2.80(\mathrm{~d}, J=3.2 \mathrm{~Hz}, 4 \mathrm{H}), 2.01$ (s, broad, $1 \mathrm{H}), 1.17(\mathrm{~s}, 3 \mathrm{H})$; ${ }^{13} \mathrm{C} \mathrm{NMR}\left(75 \mathrm{MHz}, \mathrm{CDCl}_{3}\right) \delta 212.2,202.7,137.5,130.5,128.0$, $124.5,124.4,124.1,122.02,118.20,66.9,65.6,64.1,50.3,48.1$, 30.1, 26.0, 25.9, 25.3; IR (ATR, $\mathrm{cm}^{-1}$ ): 3304, 2925, 2869, 1442, 1361, 1219; MS [EI+] m/z (\%): $391[\mathrm{M}]^{+}(10), 125\left[\mathrm{C}_{7} \mathrm{H}_{6} \mathrm{Cl}\right]^{+}$ (100). HRMS (EI): calcd. For $\mathrm{C}_{19} \mathrm{H}_{22} \mathrm{ClN}_{3} \mathrm{O}_{4}$ : 391.1299; found: 391.1303.

2.2.6. 3-\{1-[3-(2-Chlorobenzyloxy)-2-hydroxy-propyl]-[1,2,3] triazol-4-yl\}-propan-1-ol (12). Colorless oil; yield: $84 \% ;{ }^{1} \mathrm{H}$ NMR $\left(300 \mathrm{MHz}, \mathrm{CDCl}_{3}\right) \delta 7.44(\mathrm{~s}, 1 \mathrm{H}), 7.43-7.35(\mathrm{~m}, 1 \mathrm{H})$, $7.30-7.21(\mathrm{~m}, 3 \mathrm{H}), 4.78(\mathrm{~s}$, broad, $1 \mathrm{H}), 4.64(\mathrm{~s}, 2 \mathrm{H}), 4.53-4.38$ (dddd, $J=14.0,14.0,7.1,3.6 \mathrm{~Hz}), 4.28-4.20(\mathrm{~m}, 1 \mathrm{H}), 3.66(\mathrm{t}, J=$ $6.4 \mathrm{~Hz}, 2 \mathrm{H}$ ), 3.58-3.51 (dddd, $J=9.7,9.7,5.5,5.0 \mathrm{~Hz}, 2 \mathrm{H}$ ), 2.79 (t, $J=7.4 \mathrm{~Hz}, 2 \mathrm{H}), 2.17$ (s, broad, $1 \mathrm{H}), 1.89(\mathrm{p}, J=6.6 \mathrm{~Hz}, 2 \mathrm{H})$; ${ }^{13} \mathrm{C}$ NMR $\left(75 \mathrm{MHz}, \mathrm{CDCl}_{3}\right) \delta 147.0,134.8,133.1,129.3,129.2$, $126.6,122.3,71.2,70.5,68.8,61.3,52.6,31.5,21.7$; IR (ATR, $\left.\mathrm{cm}^{-1}\right): 3487,2912,2862,1716,1446,1219$; MS [EI+] $\mathrm{m} / z$ (\%): $325[\mathrm{M}]^{+}(10), 126\left[\mathrm{C}_{5} \mathrm{H}_{8} \mathrm{~N}_{3} \mathrm{O}\right]^{+}$(100). HRMS (EI): calcd. For $\mathrm{C}_{15} \mathrm{H}_{20} \mathrm{ClN}_{3} \mathrm{O}_{3}$ : 325.1193; found: 325.1195 .

2.2.7. 1-(2-Chlorobenzyloxy)-3-[4-(4-nitro-phenoxymethyl)-[1, 2,3]triazol-1-yl]-propan-2-ol (13). White solid; yield: $55 \%$; mp $85^{\circ} \mathrm{C} ;{ }^{1} \mathrm{H} \mathrm{NMR}\left(300 \mathrm{MHz}, \mathrm{CDCl}_{3}\right) \delta 8.20(\mathrm{~d}, J=8.2 \mathrm{~Hz}, 2 \mathrm{H})$, $8.17(\mathrm{~d}, J=2.1 \mathrm{~Hz}, 2 \mathrm{H}), 7.81(\mathrm{~s}, 1 \mathrm{H}), 7.43-7.23(\mathrm{~m}, 4 \mathrm{H}), 7.08$ $(\mathrm{m}, 2 \mathrm{H}), 4.63(\mathrm{~d}, J=1.1 \mathrm{~Hz}, 2 \mathrm{H}), 4.62$ (dddd, $J=14.1$, $14.07 .1,4.6 \mathrm{~Hz}, 2 \mathrm{H}), 4.30(\mathrm{~m}, 1 \mathrm{H}), 3.64-3.49$ (dddd, $J=$ 9.7, 9.7, 5.0, $4.7 \mathrm{~Hz}, 2 \mathrm{H}), 2.16(\mathrm{~s}, 1 \mathrm{H}) ;{ }^{13} \mathrm{C}$ NMR $(75 \mathrm{MHz}$, $\left.\mathrm{CDCl}_{3}\right) \delta 162.7,142.1,141.4,134.6,132.9,129.2,129.2,128.9$, $126.5,125.5,124.4,114.5,71.0,70.4,68.75,52.7 ;$ IR $\left(\right.$ ATR $\left.\mathrm{cm}^{-1}\right)$ : $3253,2878,2856,1336$; MS [EI+] $\mathrm{m} / z(\%): 418[\mathrm{M}]^{+}(5)$, $125\left[\mathrm{C}_{7} \mathrm{H}_{6} \mathrm{Cl}\right]^{+}(100)$. HRMS (EI): calcd. For $\mathrm{C}_{19} \mathrm{H}_{19} \mathrm{ClN}_{4} \mathrm{O}_{5}$ : 418.1044; found: 418.1048 .

2.2.8.1-(2-Chlorobenzyloxy)-3-[4-(4-chlorophenoxymethyl)-[1, 2,3]triazol-1-yl]-propan-2-ol (14). White solid; yield: 89\%; $\mathrm{mp} 67-68^{\circ} \mathrm{C} ;{ }^{1} \mathrm{H}$ NMR $\left(300 \mathrm{MHz}, \mathrm{CDCl}_{3}\right) \delta 7.73(\mathrm{~s}, 1 \mathrm{H})$, $7.41(\mathrm{dd}, J=9.6,4.8 \mathrm{~Hz}, 1 \mathrm{H}), 7.30-7.20(\mathrm{~m}, 5 \mathrm{H}), 6.94-6.89$ $(\mathrm{m}, 2 \mathrm{H}), 5.16(\mathrm{~s}, 2 \mathrm{H}), 4.63(\mathrm{~s}, 2 \mathrm{H}), 4.60-4.40(\mathrm{~m}, 2 \mathrm{H}), 4.29$ (dd, $J=4.5,3.7 \mathrm{~Hz}, 1 \mathrm{H}), 3.62-3.46(\mathrm{~m}, 2 \mathrm{H}), 2.95$ (s, broad, $1 \mathrm{H}) ;{ }^{13} \mathrm{C}$ NMR $\left(75 \mathrm{MHz}, \mathrm{CDCl}_{3}\right) \delta 156.1,143.0,134.3,132.8$, $128.9,128.9,128.8,128.7,128.7,126.2,123.6,115.4,70.5,70.1$, 68.5, 61.5, 52.2; IR (ATR, $\mathrm{cm}^{-1}$ ): 3168, 2921, 2869, 1489, 1237; MS [EI+] m/z (\%): $407[\mathrm{M}]^{+}(2), 185\left[\mathrm{C}_{9} \mathrm{H}_{10} \mathrm{ClO}\right]^{+}(80), 127$ $\left[\mathrm{C}_{6} \mathrm{H}_{4} \mathrm{ClO}\right]^{+}$(100). HRMS (EI): calcd. For $\mathrm{C}_{19} \mathrm{H}_{19} \mathrm{Cl}_{2} \mathrm{~N}_{3} \mathrm{O}_{3}$ : 407.0803; found: 407.0808 . 


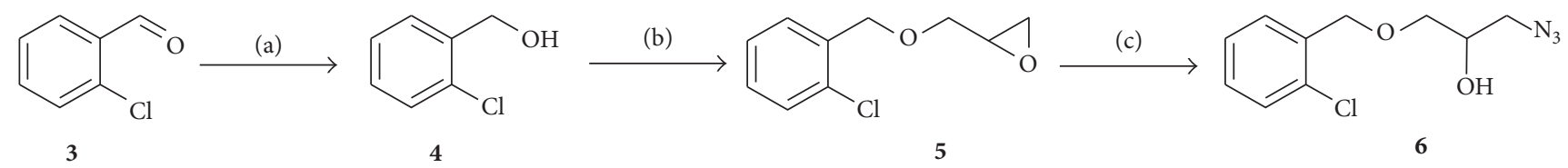

Scheme 2: Reagents and conditions: (a) $\mathrm{NaBH}_{4}$ and $\mathrm{MeOH}$. (b) Epichlorohydrin, $\mathrm{NaOH}$, and $\mathrm{N}-\mathrm{Bu}_{4} \mathrm{NBr}$. (c) $\mathrm{NaN}_{3}, \mathrm{NH}_{4} \mathrm{Cl}$, and $\mathrm{MeOH}-\mathrm{H}_{2} \mathrm{O}$.<smiles>NCC(O)COCc1ccccc1Cl</smiles>

6

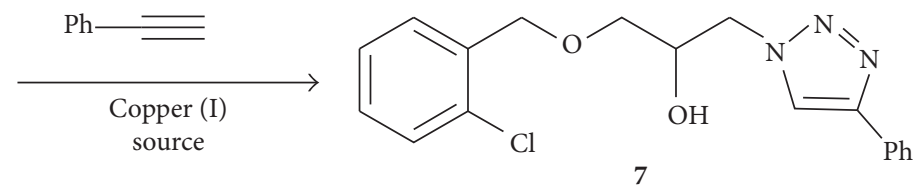

7

SCHEME 3: Reaction of azido alcohol 6 with phenylacetylene.

2.2.9. 1-(2-Chlorobenzyloxy)-3-[4-(6-methoxynaphthalen-2yl)-[1,2,3]triazol-1-yl]-propan-2-ol (15). White solid; yield: 94\%; mp $100^{\circ} \mathrm{C}$; ${ }^{1} \mathrm{H}$ NMR $\left(300 \mathrm{MHz}, \mathrm{CDCl}_{3}\right) \delta 8.10(\mathrm{~s}$, $1 \mathrm{H}), 7.90$ (s, 1H), 7.79 (dt, $J=8.5,1.8 \mathrm{~Hz}, 1 \mathrm{H}), 7.72(\mathrm{~s}, 1 \mathrm{H})$, $7.68(\mathrm{dd}, J=8.8,1.8 \mathrm{~Hz}, 1 \mathrm{H}), 7.40(\mathrm{dd}, J=17.1,9.3 \mathrm{~Hz})$, $7.29-7.20$ (m, 3H), 7.16-7.09 (m, 2H), 4.66 (s, 2H), 4.60-4.46 (ddd, $J=3.5,13.9,6.7 \mathrm{~Hz}, 2 \mathrm{H}), 4.36(\mathrm{~s}, 1 \mathrm{H}), 3.92(\mathrm{~s}, 3 \mathrm{H})$, 3.65-3.57 (dddd, $J=9.7,4.9,9.7,5.5 \mathrm{~Hz}, 2 \mathrm{H}$ ), 2.16 (s, broad, $1 \mathrm{H}) ;{ }^{13} \mathrm{C}$ NMR $\left(75 \mathrm{MHz}, \mathrm{CDCl}_{3}\right) \delta 157.9,147.6,135.1,134.3$, 133.4, 129.7, 129.6, 129.5, 129.2, 128.8, 127.3, 126.9, 125.5, 124.9, 121.2, 119.2, 105.7, 71.5, 70.8, 69.2, 55.3, 53.3; IR (ATR, $\left.\mathrm{cm}^{-1}\right)$ : 3391, 3131, 2921, 2852, 1606, 1216; MS [EI+] $\mathrm{m} / z$ (\%): 423 $[\mathrm{M}]^{+}(10), 226\left[\mathrm{C}_{13} \mathrm{H}_{10} \mathrm{~N}_{3} \mathrm{O}\right]^{+}(80), 185\left[\mathrm{C}_{9} \mathrm{H}_{10} \mathrm{ClO}\right]^{+}$(35), $125\left[\mathrm{C}_{7} \mathrm{H}_{6} \mathrm{Cl}\right]^{+}$(100). HRMS (EI): calcd. For $\mathrm{C}_{23} \mathrm{H}_{22} \mathrm{ClN}_{3} \mathrm{O}_{3}$ : 423.1350; found: 423.1356 .

2.2.10. 1-(2-Chlorobenzyloxy)-3-[4-(4-fluorophenyl)-[1,2,3]triazol-1-yl]-propan-2-ol (16). Colorless oil; yield: $66 \% ;{ }^{1} \mathrm{H}$ $\operatorname{NMR}\left(300 \mathrm{MHz}, \mathrm{CDCl}_{3}\right) \delta 7.82(\mathrm{~s}, 1 \mathrm{H}), 7.77-7.70(\mathrm{~m}, 2 \mathrm{H}), 7.53$ $(\mathrm{dd}, J=5.7,3.3 \mathrm{~Hz}, 1 \mathrm{H}), 7.38(\mathrm{td}, J=7.4,2.2 \mathrm{~Hz}, 1 \mathrm{H}), 7.26$ (ddt, $J=7.6,2.2,1.1 \mathrm{~Hz}, 1 \mathrm{H}), 7.30-7.21(\mathrm{~m}, 2 \mathrm{H}), 7.09(\mathrm{t}, J=7.4 \mathrm{~Hz}$, $1 \mathrm{H}), 4.65(\mathrm{~d}, J=0.8 \mathrm{~Hz}, 2 \mathrm{H}), 4.61-4.46(\mathrm{dd}, J=14.0,7.0 \mathrm{~Hz}$, $2 \mathrm{H}), 4.31(\mathrm{p}, J=5.2 \mathrm{~Hz}, 1 \mathrm{H}), 3.64-3.54(\mathrm{dd}, J=9.7,5.7 \mathrm{~Hz}$, 2H), $2.17(\mathrm{~s}, 1 \mathrm{H}) ;{ }^{13} \mathrm{C} \mathrm{NMR}\left(75 \mathrm{MHz}, \mathrm{CDCl}_{3}\right) \delta 164.1,160.8$, $146.5,134.8,133.2,129.5,129.4,129.1,127.2,127.1,126.7,126.4$, $120.8,115.7,115.5,71.2,70.6,69.0,52.9$; IR (ATR, $\left.\mathrm{cm}^{-1}\right): 3365$, 2922, 2864, 1497, 1224; MS [EI+] $m / z(\%): 361[\mathrm{M}]^{+}$(25), 164 $\left[\mathrm{C}_{8} \mathrm{H}_{7} \mathrm{FN}_{3}\right]^{+}$(100). HRMS (EI): calcd. For $\mathrm{C}_{18} \mathrm{H}_{17} \mathrm{ClFN}_{3} \mathrm{O}_{2}$ : 361.0993; found: 361.0997.

2.2.11. 1-(2-Chlorobenzyloxy)-3-[4-(2,4-difluorophenyl)-[1,2,3] triazol-1-yl]-propan-2-ol (17). White solid; yield: 67\%; mp $77^{\circ} \mathrm{C}$; ${ }^{1} \mathrm{H}$ NMR $\left(300 \mathrm{MHz}, \mathrm{CDCl}_{3}\right) \delta 8.31-8.15(\mathrm{~m}, 1 \mathrm{H})$, $8.00(\mathrm{~s}, 1 \mathrm{H}), 7.47-7.33(\mathrm{~m}, 2 \mathrm{H}), 7.26(\mathrm{dd}, J=5.9,3.5 \mathrm{~Hz}$, $2 \mathrm{H}), 6.99(\mathrm{td}, J=8.4,2.6 \mathrm{~Hz}, 1 \mathrm{H}), 6.88(\mathrm{ddd}, J=11.1,8.9$, $2.6 \mathrm{~Hz}, 1 \mathrm{H}), 4.66(\mathrm{~s}, 2 \mathrm{H}), 4.61-4.49$ (ddd, $J=14.1,6.9,3.7 \mathrm{~Hz}$, $2 \mathrm{H}$ ), 4.37-4.27 (m, 1H), 3.65-3.55 (dddd, $J=9.7,9.6,5.6$, $4.6 \mathrm{~Hz}, 2 \mathrm{H}), 1.65$ (s, broad, $1 \mathrm{H}) ;{ }^{13} \mathrm{C} \mathrm{NMR}\left(75 \mathrm{MHz}, \mathrm{CDCl}_{3}\right)$ $\delta 163.4,160.1,139.6,134.9,134.3,132.6,128.8,128.5,126.1$, $123.21,114.2,111.1,103.6,70.74,70.1,68.5,52.4$; IR (ATR, $\left.\mathrm{cm}^{-1}\right): 3194,3151,2908,1361 ; \mathrm{MS}[\mathrm{EI}+] \mathrm{m} / z(\%): 379[\mathrm{M}]^{+}$ (10), $239\left[\mathrm{C}_{10} \mathrm{H}_{11} \mathrm{~F}_{2} \mathrm{~N}_{3} \mathrm{O}\right]^{+}$(100). HRMS (EI): calcd. For $\mathrm{C}_{18} \mathrm{H}_{16} \mathrm{ClF}_{2} \mathrm{~N}_{3} \mathrm{O}_{2}$ : 379.0889; found: 379.0891 .
2.2.12. 1-(2-Chlorobenzyloxy)-3-[4-(2-trifluoromethylphenyl)[1,2,3]triazol-1-yl]-propan-2-ol (18). Colorless oil; yield: 68\%; ${ }^{1} \mathrm{H}$ NMR $\left(300 \mathrm{MHz}, \mathrm{CDCl}_{3}\right) \delta 7.91(\mathrm{~s}, 1 \mathrm{H}), 7.90(\mathrm{~d}, J=6.9 \mathrm{~Hz}$, $1 \mathrm{H}), 7.73(\mathrm{dd}, J=8.0,1.4 \mathrm{~Hz}, 1 \mathrm{H}), 7.47(\mathrm{dd}, J=7.9,1.5 \mathrm{~Hz}, 1 \mathrm{H})$, $7.42(\mathrm{ddd}, J=6.9,3.7,1.6 \mathrm{~Hz}, 1 \mathrm{H}), 7.37-7.32(\mathrm{~m}, 1 \mathrm{H}), 7.23(\mathrm{td}, J=$ 6.5, 5.7, 3.7 Hz, 2H), 4.63 (d, $J=1.2 \mathrm{~Hz}, 2 \mathrm{H}$ ), 4.63-4.50 (dddd, $J=14.1,14.0,6.8,3.9 \mathrm{~Hz}, 2 \mathrm{H}), 4.30(\mathrm{dq}, J=9.8,5.3 \mathrm{~Hz}, 1 \mathrm{H})$, 3.60-3.51 (dddd, $J=9.8,9.7,5.6,4.9 \mathrm{~Hz}, 2 \mathrm{H}$ ), 1.26 (s, broad, $1 \mathrm{H}) ;{ }^{13} \mathrm{C}$ NMR $\left(75 \mathrm{MHz}, \mathrm{CDCl}_{3}\right) \delta 163.4,160.1,139.6,134.9$, $134.3,132.6,128.8,128.5,126.1,123.21,114.2,111.1,103.6,70.74$, 70.1, 68.5, 52.4; IR (ATR, $\mathrm{cm}^{-1}$ ): 3344, 1354, 1168.86, 1107; MS $[\mathrm{EI}+] \mathrm{m} / z(\%): 411[\mathrm{M}]^{+}(5), 125\left[\mathrm{C}_{7} \mathrm{H}_{6} \mathrm{Cl}\right]^{+}(100)$. HRMS (EI): calcd. For $\mathrm{C}_{19} \mathrm{H}_{17} \mathrm{ClF}_{3} \mathrm{~N}_{3} \mathrm{O}_{2}$ : 411.0961; found: 411.0965.

2.3. Theoretical Calculations. All the electronic structure computations were carried out with NWChem package [14]. A frequency analysis was applied to the optimized geometries to verify the corresponding stability criterion. The PBEPBE [15] exchange and correlation functional was used with the 6$311++g^{* *}$ Pople's basis set. All the reactivity parameters were computed from a finite differences approach.

\section{Results and Discussion}

3.1. Chemistry. The first experiments were carried out with the objective to prepare 1-azido-3-(2-chloro-benzyl-oxy)-propan-2-ol, 6 (Scheme 2). Initially, 2-chlorophenyl methanol 4 prepared from reduction of 4-chlorobenzaldehyde 3 was reacted with epichlorohydrin to give the aryl epoxide $\mathbf{5}$ $[9,15]$ which was opened with sodium azide to afford azido alcohol 6 in a $62 \%$ global yield from 4-chlorobenzaldehyde 3 .

Azido alcohol 6 was the starting material for the following CuAAC experiments. In a model reaction, compound $\mathbf{6}$ was treated with phenylacetylene in presence of diverse $\mathrm{Cu}(\mathrm{I})$ catalytic sources according to that described by Rostovtsev et al. [16], Tornøe et al. [17] and our group [18, 19] (Scheme 3), obtaining compound 7 as only reaction product. From these experiments presented in Table 1, we selected CuI-DIPEA for $\mathrm{CuAAC}$ reaction because a best yield (85\%) was reached using this catalytic system.

Taking these optimized conditions, a synthetic procedure was developed and a series of (2-chlorobenzyloxy)-1,2,3triazolyl-propan-2-ol derivatives was prepared from azido 
TABLE 1: Effect of catalyst, reducing agent, base, and solvent.

\begin{tabular}{|c|c|c|c|c|c|}
\hline Entry & Copper salt & Reducing agent & Base & Solvent & $\%$ yield \\
\hline 7 & $\mathrm{CuSO}_{4}$ & Glucose & $\mathrm{NaOH}$ & $\mathrm{MeOH}-\mathrm{H}_{2} \mathrm{O}$ & 34 \\
\hline 8 & $\mathrm{CuSO}_{4}$ & Sodium ascorbate & none & $\mathrm{tBuOH}-\mathrm{H}_{2} \mathrm{O}$ & 28 \\
\hline 9 & $\mathrm{CuI}$ & None & $\mathrm{NaOH}$ & $\mathrm{MeOH}-\mathrm{H}_{2} \mathrm{O}$ & 40 \\
\hline 10 & $\mathrm{CuI}$ & None & DIPEA & $\mathrm{MeOH}$ & 35 \\
\hline 11 & $\mathrm{CuI}$ & None & DIPEA & $\mathrm{CH}_{2} \mathrm{Cl}_{2}$ & 85 \\
\hline
\end{tabular}

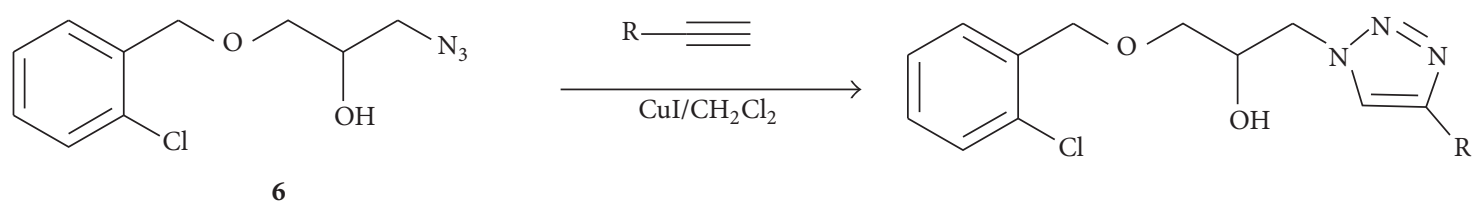

SCHEMe 4

TABLE 2: 1,2,3-Triazoles prepared via CuAAC.

\begin{tabular}{|c|c|c|}
\hline Compound & $\mathrm{R}^{1}$ & $\%$ yield \\
\hline 7 & $\mathrm{Ph}$ & 85 \\
\hline 8 & $\mathrm{CH}_{2} \mathrm{O}\left(4-\mathrm{CH}_{3}\right) \mathrm{C}_{6} \mathrm{H}_{4}$ & 99 \\
\hline 9 & $\mathrm{CH}_{2} \mathrm{O}\left(4-\mathrm{OCH}_{3}\right) \mathrm{C}_{6} \mathrm{H}_{4}$ & 46 \\
\hline 10 & $\begin{array}{c}4-\mathrm{CH}_{3} \mathrm{C}_{6} \mathrm{H}_{4} \\
\mathrm{O} \\
\text { II }\end{array}$ & 51 \\
\hline 11 & $\mathrm{O}$ & 66 \\
\hline 12 & $\left(\mathrm{CH}_{2}\right)_{3} \mathrm{OH}$ & 84 \\
\hline 13 & $\mathrm{CH}_{2} \mathrm{O}\left(4-\mathrm{NO}_{2}\right) \mathrm{C}_{6} \mathrm{H}_{4}$ & 55 \\
\hline 14 & $\mathrm{CH}_{2} \mathrm{O}(4-\mathrm{Cl}) \mathrm{C}_{6} \mathrm{H}_{4}$ & 89 \\
\hline 15 & & 94 \\
\hline 16 & $4-\mathrm{FC}_{6} \mathrm{H}_{4}$ & 66 \\
\hline 17 & $2,4-\mathrm{FC}_{6} \mathrm{H}_{3}$ & 67 \\
\hline 18 & $2-\mathrm{CF}_{3} \mathrm{C}_{6} \mathrm{H}_{4}$ & 68 \\
\hline
\end{tabular}

alcohol 6 with different alkynes. The results in Table 2 show that this process occurs with high efficiency and functional group tolerance; see Scheme 4.

3.2. DFT Characterization. In order to compare triazoles 7-18 with compound $\mathbf{1}$ and to establish possible SSRI candidates, a theoretical study was carried out. The global reactivity indexes of studied compounds are presented in Table 3, electronic affinity $(A)$, ionization potential $(I)$, chemical potential $(\mu)$, chemical softness $(S)$, and electrophilicity $(\omega)$. The obtained results are gathered in Table 3.

Ionization potential $(I)$ is defined as the energy needed to remove an electron from a molecule. According to obtained results, the molecule 12 showed the highest ionization potential value, and the molecule $\mathbf{1 5}$ has the closest value to $\mathbf{1}$, showing that electron-rich aromatic rings are necessary to mimic the properties of molecule 1 . On the other hand, the presence of electron attracting groups in the aromatics increases the value of $I$; the aliphatic moieties have the same effect.

Electron affinity (EA) measures the ability of a molecule to accept electrons and form anions. There are some EA negative in the obtained values, because the molecules released energy in the process of electron acceptance. In a general view, only the half of the molecules has positive EA values like one. Nevertheless, the computed values are different compared with 1, with the molecules 16 with a behavior closest to $\mathbf{1}$.

Chemical potential $(\mu)$ measures the escaping tendency of an electronic cloud in the ground state system. The obtained results shown that molecule $\mathbf{1}$ has higher value (less negative) than synthesized triazolyl derivatives, making compound 1 a better electron donor than obtained compounds. Nevertheless, almost all the compounds have pretty close values to $\mathbf{1}$, except for compound 7 and in minor degree $\mathbf{1 2 .}$

Chemical softness $(S)$ is related to polarizability. The values $S$ for compounds $\mathbf{1 4}$ and $\mathbf{1 8}$ are remarkably close to the value of compound $\mathbf{1}$, suggesting a similar facility to charge transfer; other compounds with similar values are 15-17, respectively. As matter of fact, the trifluorobenzyl group in compound 18 shows similar characteristics to indolyl moiety in compound $\mathbf{1}$.

Electrophilicity $(\omega)$ gives an idea of the stabilization energy when the system acquires electrons from the environment up to saturation. Since the electrophilicity power is computed through $\left(\mu^{2} / 2 \eta\right)$, the differences in electrophilicity power $(\omega)$ between the compounds are mainly due to differences in their chemical potential. In this regard, the results suggest that triazole moiety is a better nucleophile for compounds 7-18 than tetrahydropyridine system in compound 1 . In a general way all the parameters obtained by DFT calculations show the necessity of having an electronrich aromatic bond to mimic the indolyl moiety in compound 1 . 
TABLE 3: Global reactivity indexes in $\mathrm{eV}$.

\begin{tabular}{|c|c|c|c|c|c|}
\hline & $A$ & $I$ & $\mu$ & $S$ & $\omega$ \\
\hline 1 & 0.113 & 6.546 & -3.216 & 0.155 & 0.804 \\
\hline 7 & -1.042 & 7.717 & -8.879 & 0.056 & 2.219 \\
\hline 8 & -0.162 & 7.593 & -3.878 & 0.128 & 0.969 \\
\hline 9 & -0.129 & 7.250 & -3.689 & 0.135 & 0.922 \\
\hline 10 & -0.105 & 7.510 & -3.808 & 0.131 & 0.952 \\
\hline 12 & -0.158 & 8.362 & -4.260 & 0.117 & 1.065 \\
\hline 13 & 0.911 & 8.253 & -3.671 & 0.136 & 0.917 \\
\hline 14 & 0.347 & 7.312 & -3.482 & 0.143 & 0.870 \\
\hline 15 & 0.526 & 6.619 & -3.044 & 0.164 & 0.761 \\
\hline 16 & 0.271 & 7.397 & -3.562 & 0.140 & 0.890 \\
\hline 17 & 0.371 & 7.537 & -3.583 & 0.139 & 0.895 \\
\hline 18 & 0.568 & 7.688 & -3.559 & 0.140 & 0.889 \\
\hline
\end{tabular}

$\mu=1 / 2\left(\varepsilon_{I}+\varepsilon_{A}\right) ; \eta=\varepsilon_{A}-\varepsilon_{I} ; S=1 / ; \omega=\mu^{2} / 2 \eta$.

TABLE 4: Local reactivity indexes for selected atoms.

\begin{tabular}{|c|c|c|c|c|c|}
\hline Compound & & $f^{+}$ & $f^{-}$ & $s^{+}$ & $s^{-}$ \\
\hline \multirow{3}{*}{7} & N1 & 0.01 & 0.04 & 0.01 & 0.06 \\
\hline & $\mathrm{N} 2$ & 0.02 & 0.08 & 0.03 & 0.12 \\
\hline & N3 & 0.02 & 0.04 & 0.03 & 0.06 \\
\hline \multirow{3}{*}{8} & N1 & 0.00 & 0.02 & 0.00 & 0.07 \\
\hline & $\mathrm{N} 2$ & 0.01 & 0.03 & 0.03 & 0.10 \\
\hline & N3 & 0.02 & 0.01 & 0.07 & 0.03 \\
\hline \multirow{3}{*}{9} & N1 & 0.00 & 0.04 & 0.00 & 0.14 \\
\hline & N2 & 0.02 & 0.08 & 0.07 & 0.29 \\
\hline & N3 & 0.01 & 0.04 & 0.03 & 0.14 \\
\hline \multirow{3}{*}{10} & N1 & 0.01 & 0.04 & 0.03 & 0.14 \\
\hline & N2 & 0.02 & 0.08 & 0.07 & 0.28 \\
\hline & N3 & 0.02 & 0.03 & 0.07 & 0.10 \\
\hline \multirow{3}{*}{12} & N1 & 0.00 & 0.03 & 0.00 & 0.11 \\
\hline & $\mathrm{N} 2$ & 0.01 & 0.10 & 0.03 & 0.37 \\
\hline & N3 & 0.01 & 0.04 & 0.03 & 0.14 \\
\hline \multirow{3}{*}{13} & N1 & 0.01 & 0.03 & 0.04 & 0.12 \\
\hline & $\mathrm{N} 2$ & 0.02 & 0.06 & 0.08 & 0.23 \\
\hline & N3 & 0.00 & 0.03 & 0.00 & 0.12 \\
\hline \multirow{3}{*}{14} & N1 & 0.02 & 0.01 & 0.08 & 0.13 \\
\hline & $\mathrm{N} 2$ & 0.05 & 0.03 & 0.22 & 0.27 \\
\hline & N3 & 0.03 & 0.02 & 0.13 & 0.13 \\
\hline \multirow{3}{*}{15} & N1 & 0.01 & 0.03 & 0.04 & 0.11 \\
\hline & N2 & 0.03 & 0.05 & 0.11 & 0.19 \\
\hline & N3 & 0.02 & 0.03 & 0.07 & 0.11 \\
\hline \multirow{3}{*}{16} & N1 & 0.02 & 0.02 & 0.07 & 0.07 \\
\hline & N2 & 0.02 & 0.03 & 0.07 & 0.11 \\
\hline & N3 & 0.03 & 0.04 & 0.11 & 0.15 \\
\hline \multirow{3}{*}{17} & N1 & 0.00 & 0.04 & 0.00 & 0.15 \\
\hline & $\mathrm{N} 2$ & 0.02 & 0.07 & 0.07 & 0.27 \\
\hline & N3 & 0.02 & 0.04 & 0.07 & 0.17 \\
\hline \multirow{3}{*}{18} & N1 & 0.02 & 0.02 & 0.08 & 0.08 \\
\hline & N2 & 0.02 & 0.04 & 0.08 & 0.16 \\
\hline & N3 & 0.03 & 0.03 & 0.12 & 0.12 \\
\hline
\end{tabular}




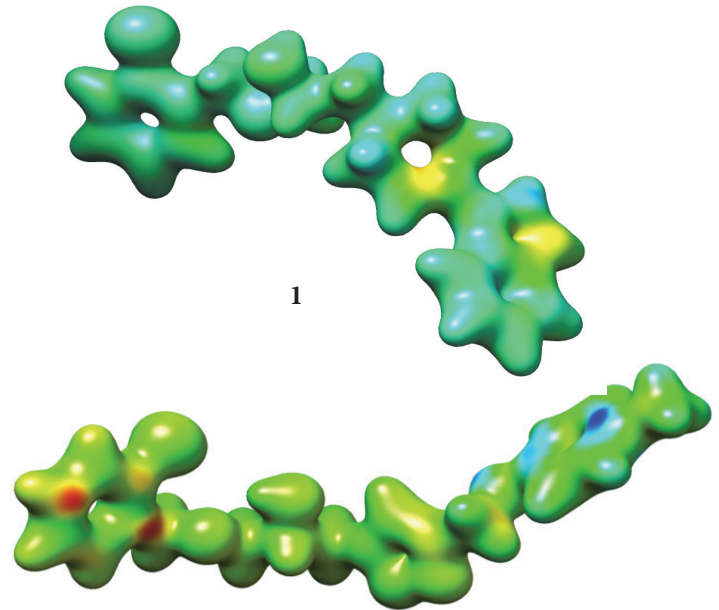

8

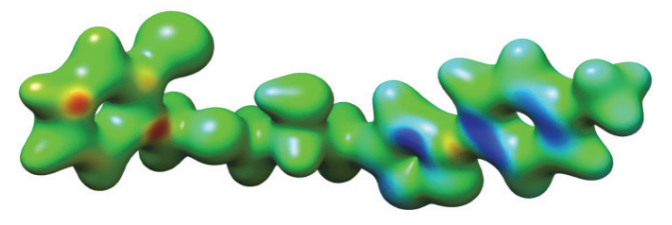

10

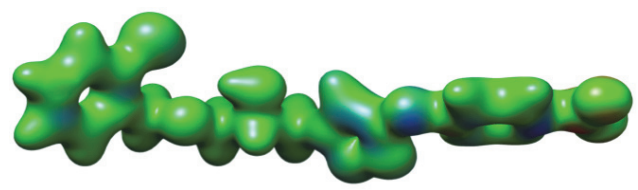

13

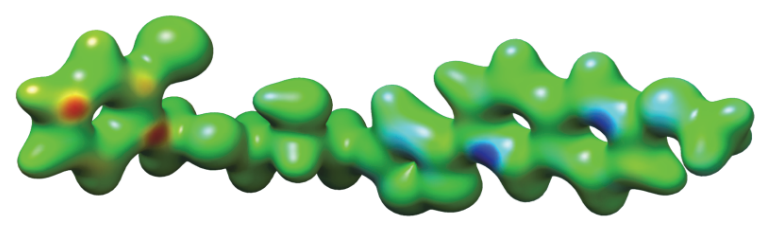

15

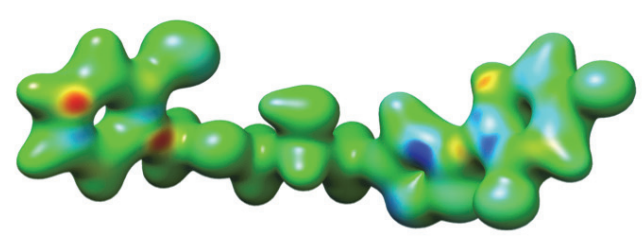

17

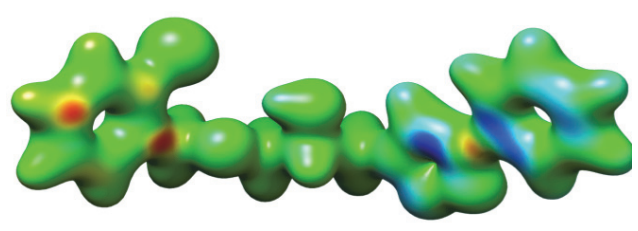

7

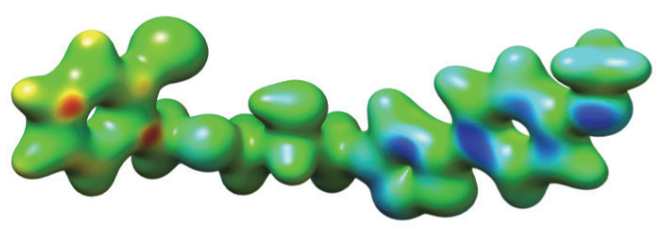

9

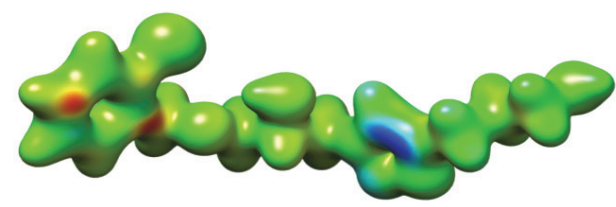

12

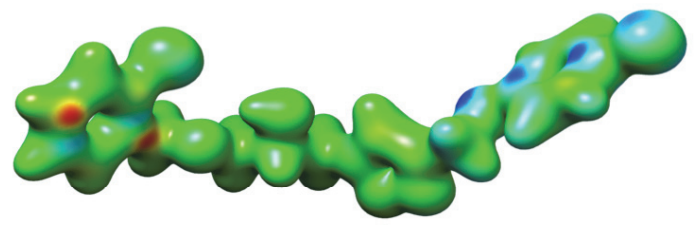

14

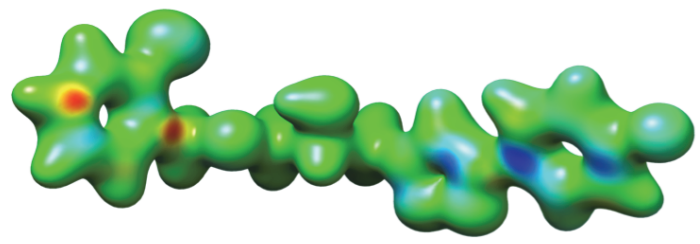

16

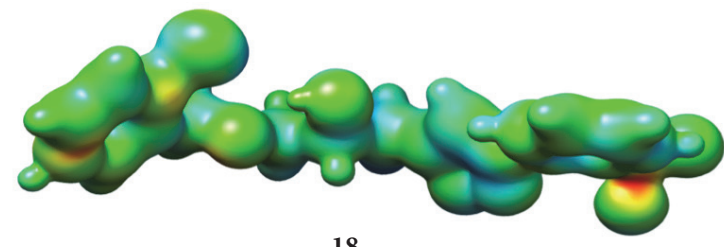

18

Figure 1: Dual descriptor $f^{ \pm}$for the molecules studied in this work.

The local properties of synthesized compounds are hard to analyze due to the diverse nature of substituent groups used in this study. For this reason, the three nitrogen atoms in triazole ring were used as a probe for the local properties in the prepared molecules. As shown in Table 4, the highest values of $f^{-}$can be observed for $\mathrm{N} 2$ atom in all molecules, indicating that this atom is the most susceptible to a nucleophilic attack. In addition, a visible tendency for the case of $f^{+}$was not found. The local softness $(s)$ followed the same tendencies as Fukui functions; nevertheless, this function could be comparable between molecules, being higher in compounds $\mathbf{1 4}-\mathbf{1 8}$ on N2 atom. 
Other important index is known as dual descriptor $f^{ \pm}$ which contains information about the chemical philicity (electrophilicity/nucleophilicity). In this case, the graphic maps are generally sufficient for rationalizing the chemical reactivity. The maps of dual descriptor for the set of molecules studied in this work are represented in Figure 1, displaying the most probable sites for a possible nucleophilic-electrophilic attack. In a general way, the most electrophilic sites are found on the chlorobenzyl moiety, while the most nucleophilic sites are located on the opposite side of the molecule independent of the electronic nature of substituent group. These elements reveal some similarities between compound $\mathbf{1}$ and synthesized compounds with nearest values for compounds 14-18. The calculated parameters also allow establishing interaction sites in these molecules.

\section{Conclusions}

A new class of derivatives of chlorobenzyloxy triazolylpropan-2-ol was obtained from CuAAC reaction using commercially available raw materials through simple and efficient methods. Theoretical calculations indicate that a high similarity can be found between these products and the leading molecule 1; consequently, a good SSRI ability would be expected for these compounds, suggesting more studies about this topic. Also, DFT descriptors show that the presence of an electron-rich aromatic ring is necessary to mimic the indolyl moiety present in compound $\mathbf{1}$.

\section{Conflicts of Interest}

The authors declare that they have no conflicts of interest.

\section{Acknowledgments}

Financial support from CONACYT (Project no. 135053) is gratefully acknowledged. The authors would like to thank Signa S. A. de C. V. for some graciously donated solvents and reagents and to N. Zavala, A. Nuñez, and L. Triana for the technical support.

\section{References}

[1] M. Reddy, "Depression: the disorder and the burden," Indian Journal of Psychological Medicine, vol. 32, no. 1, pp. 1-2, 2010.

[2] M. Marcus, M. T. Yasamy, M. Ommeren, D. Chisholm, and S. Saxena, Depression: A Global Public Health Concern, World Health Organization, 2012, http://www.who.int/mental_health/ management/depression/who_paper_depression_wfmh_2012 .pdf.

[3] K. Sangkuhl, T. Klein, and R. Altman, "Selective Serotonin Reuptake Inhibitors (SSRI) pathway," Pharmacogenetics Genomics, vol. 19, no. 11, pp. 907-909, 2009.

[4] M. Vaswani, F. K. Linda, and S. Ramesh, "Role of selective serotonin reuptake inhibitors in psychiatric disorders: a comprehensive review," Progress in Neuro-Psychopharmacology and Biological Psychiatry, vol. 27, no. 1, pp. 85-102, 2003.

[5] D. J. Nutt, S. Forshall, C. Bell et al., "Mechanisms of action of selective serotonin reuptake inhibitors in the treatment of psychiatric disorders," European Neuropsychopharmacology, vol. 9, supplement 3, pp. S81-S86, 1999.

[6] O. M. Ghoneim, D. A. Ibrahim, I. M. El-Deeb, S. H. Lee, and R. G. Booth, "A novel potential therapeutic avenue for autism: design, synthesis and pharmacophore generation of SSRIs with dual action," Bioorganic and Medicinal Chemistry Letters, vol. 21, no. 22, pp. 6714-6723, 2011.

[7] J. I. Andrés, J. Alcázar, J. M. Alonso et al., "Synthesis of 7-amino-3a,4-dihydro-3H-[1]benzopyrano[4,3-c]isoxazole derivatives displaying combined $\alpha 2$-adrenoceptor antagonistic and 5-HT reuptake inhibiting activities," Bioorganic and Medicinal Chemistry, vol. 14, no. 13, pp. 4361-4372, 2006.

[8] J. M. Dorsey, M. G. Miranda, N. V. Cozzi, and K. G. Pinney, "Synthesis and biological evaluation of 2-(4-fluorophenoxy)2-phenyl-ethyl piperazines as serotonin-selective reuptake inhibitors with a potentially improved adverse reaction profile," Bioorganic and Medicinal Chemistry, vol. 12, no. 6, pp. 14831491, 2004.

[9] T. L. Nolan, D. J. Lapinsky, J. N. Talbot et al., "Identification of a novel selective serotonin reuptake inhibitor by coupling monoamine transporter-based virtual screening and rational molecular hybridization," ACS Chemical Neuroscience, vol. 2, no. 9, pp. 544-552, 2011.

[10] J. Barluenga, F. Aznar, C. Valdés, and M.-P. Cabal, “Stereoselective synthesis of 4-piperidone and 4-aminotetrahydropyridine derivatives by the imino Diels-Alder reaction of 2-amino-1,3butadienes," Journal of Organic Chemistry, vol. 58, no. 12, pp. 3391-3396, 1993.

[11] M. M. Faul, M. E. Kobierski, and M. E. Kopach, "Green chemistry approach to the synthesis of N-substituted piperidones," Journal of Organic Chemistry, vol. 68, no. 14, pp. 5739-5741, 2003.

[12] P. Geerlings, F. De Proft, and W. Langenaeker, "Conceptual density functional theory," Chemical Reviews, vol. 103, no. 5, pp. 1793-1873, 2003.

[13] F. Aparicio, N. González-Rivas, J. Ireta et al., "Soft-Soft interactions in the protein-protein recognition process: the $\mathrm{K}^{+}$ channel-charybdotoxin case," International Journal of Quantum Chemistry, vol. 112, no. 22, pp. 3618-3623, 2012.

[14] E. Apra, T. L. Windus, T. P. Straatsma et al., NWChem, A Computational Chemistry Package for Parallel Computers, Version 5.1, Pacific Northwest National Laboratory, Richland, Wash, USA, 2005.

[15] C. Adamo and V. Barone, "Toward reliable density functional methods without adjustable parameters: the PBE0 model," Journal of Chemical Physics, vol. 110, no. 13, pp. 6158-6170, 1999.

[16] V. V. Rostovtsev, L. G. Green, V. V. Fokin, and K. B. Sharpless, "A stepwise huisgen cycloaddition process: copper(I)-catalyzed regioselective 'ligation' of azides and terminal alkynes," Angewandte Chemie - International Edition, vol. 41, no. 14, pp. 2596$2599,2002$.

[17] C. W. Tornøe, C. Christensen, and M. Meldal, "Peptidotriazoles on solid phase: [1,2,3]-Triazoles by regiospecific copper(I)catalyzed 1,3-dipolar cycloadditions of terminal alkynes to azides," Journal of Organic Chemistry, vol. 67, no. 9, pp. 30573064, 2002.

[18] M. A. García, Z. G. Ríos, J. González et al., “The use of glucose as alternative reducing agent in copper-catalyzed alkyne-azide cycloaddition," Letters in Organic Chemistry, vol. 8, no. 10, pp. 701-706, 2011. 
[19] J. González, V. M. Pérez, D. O. Jiménez, G. Lopez-Valdez, D. Corona, and E. Cuevas-Yañez, "Effect of temperature on triazole and bistriazole formation through copper-catalyzed alkyneazide cycloaddition," Tetrahedron Letters, vol. 52, no. 27, pp. 3514-3517, 2011. 

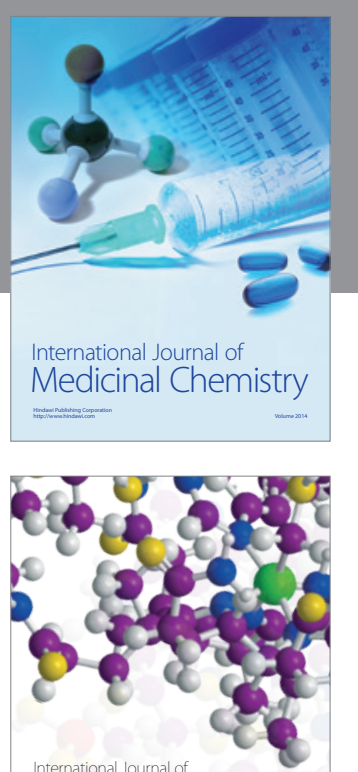

Carbohydrate Chemistry

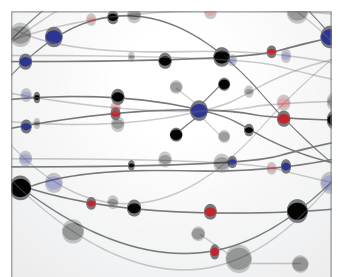

The Scientific World Journal
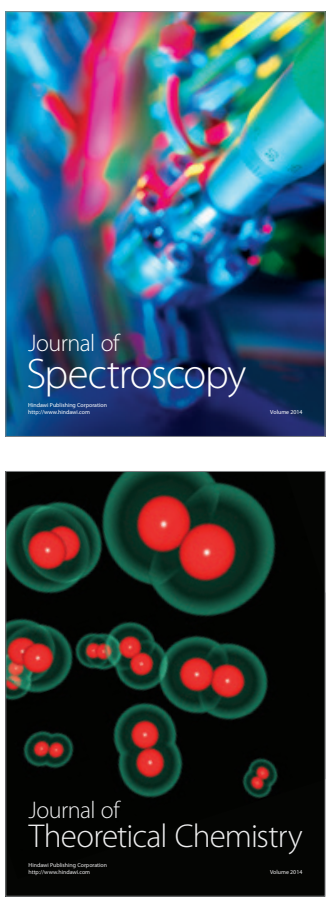
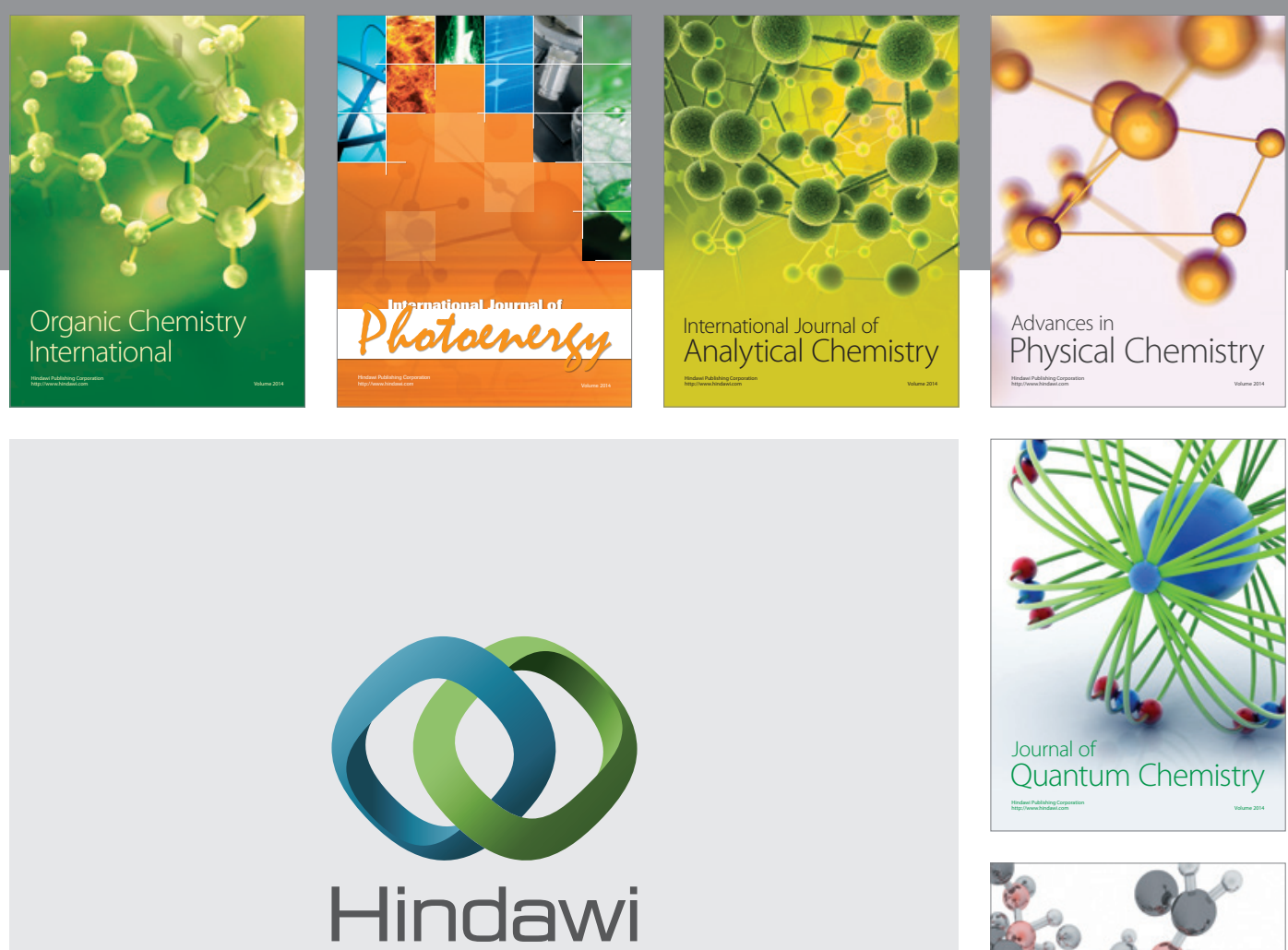

Submit your manuscripts at

https://www.hindawi.com

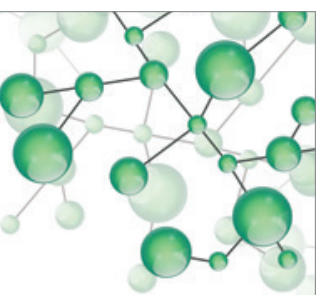

International Journal of

Inorganic Chemistry
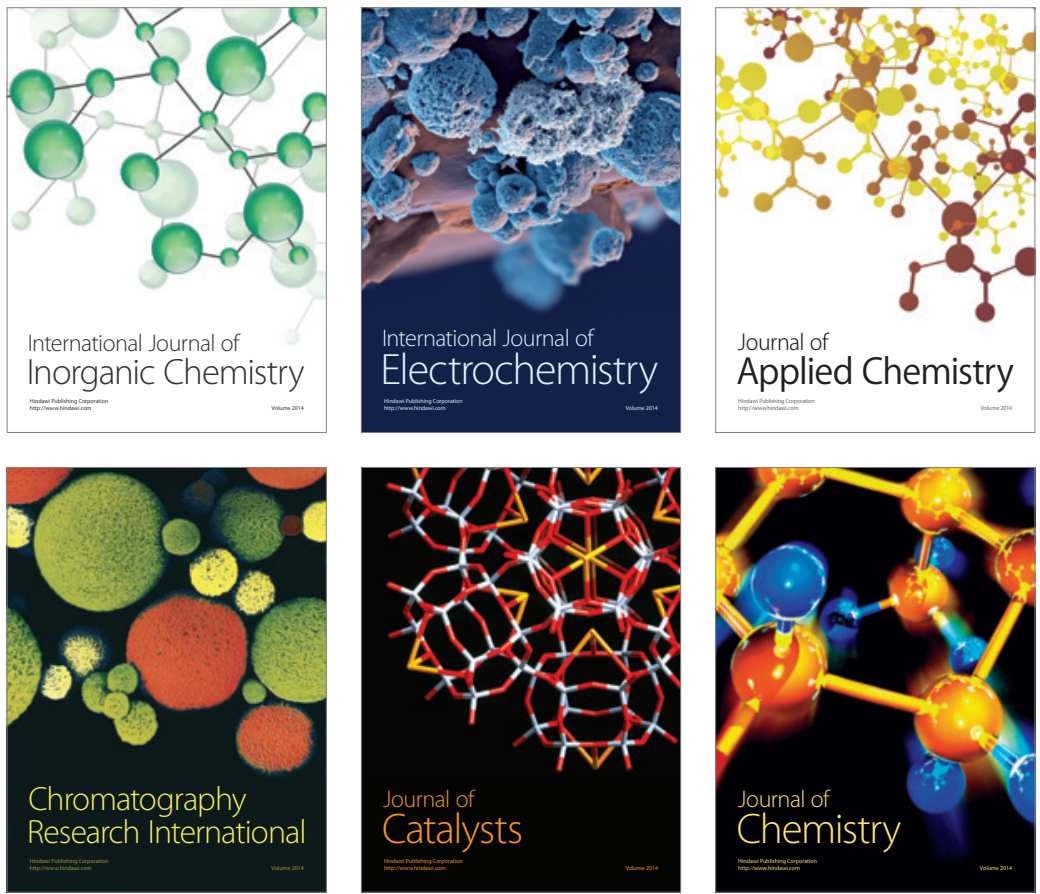

Journal of

Applied Chemistry
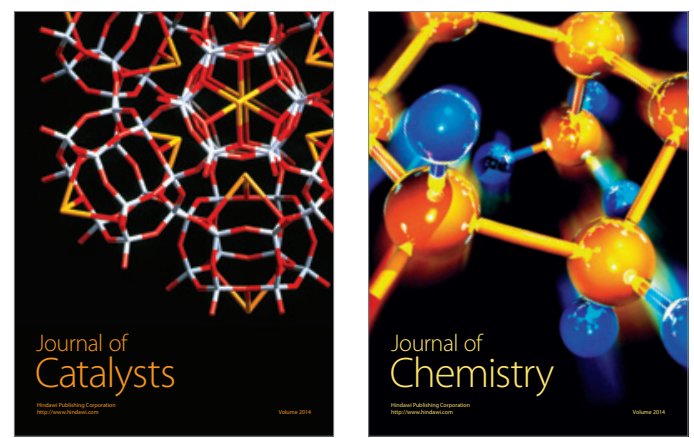
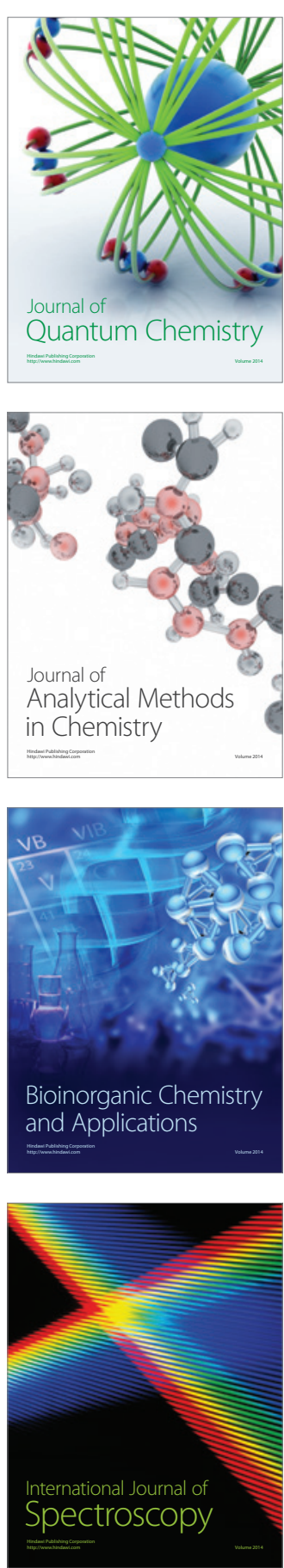